\title{
Melanonychia and mucocutaneous hyperpigmentation from hydroxyurea use for the treatment of essential thrombocytosis
}

\author{
Suman S Karanth${ }^{1}, \mathrm{MD}$, Anurag Gupta ${ }^{2}, \mathrm{MCh}$, Mukhyaprana Prabhu${ }^{1}$, MD
}

\begin{abstract}
Hydroxyurea is an antineoplastic agent commonly used to treat essential thrombocytosis. We report the case of a 50-year-old woman who was incidentally detected to have essential thrombocytosis after suffering an episode of cerebrovascular accident with faciobrachial monoparesis. She was subsequently initiated on hydroxyurea. Within seven weeks of therapy, the patient noticed irregular hyperpigmented patches over her feet, hands and perioral region, with bluish-grey longitudinal bands on all 20 nails. Hydroxyurea-induced hyperpigmentation and melanonychia are not commonly reported. To the best of our knowledge, this is only the third published report of hydroxyurea-induced hyperpigmentation and melanonychia involving all 20 nails. Physicians need to be aware of such mucocutaneous side effects to avoid misdiagnosis and unwarranted fear in patients. The decision to discontinue the intake of the drug depends heavily on the future risk of thrombotic events.
\end{abstract}

Keywords: essential thrombocytosis, hydroxyurea, hyperpigmentation, melanonychia

\section{INTRODUCTION}

Hydroxyurea is an antineoplastic agent that inhibits deoxyribonucleic acid synthesis. It is commonly used for the treatment of myeloproliferative diseases such as chronic myeloid leukaemia and essential thrombocytosis. ${ }^{(1)}$ While cases of hydroxyurea-induced hyperpigmentation have been reported, reports of hydroxyurea-induced melanonychia are uncommon, with only two such cases involving all 20 nails. ${ }^{(2)}$ Herein, we present one such rare scenario, where a patient developed hydroxyurea-induced hyperpigmentation and melanonychia within seven weeks after the initiation of therapy.

\section{CASE REPORT}

A 50-year-old Indian woman with essential thrombocytosis complained of progressive darkening of the nails of both her hands and feet. Four months prior to this, she had suffered from right faciobrachial monoparesis. Magnetic resonance imaging of the patient's brain at that time had shown a right basal ganglia infarct. The patient recovered completely, with no residual neurological deficits. Laboratory findings were significant for elevated platelet count, which was at 1,743 $\times 10^{9} / \mathrm{L}$ (normal range 150-400 $\times 10^{9} / \mathrm{L}$ ). Bone marrow aspiration and biopsy confirmed essential thrombocytosis. The patient was thus started on hydroxyurea at a dose of $1 \mathrm{~g} /$ day. She did not suffer from any side effects at the start of therapy. However, at follow-up seven weeks later, the patient complained of pigmentation involving all her fingernails and toenails.

Physical examination revealed bluish-grey longitudinal bands at the proximal aspect of the nail beds with distal progression

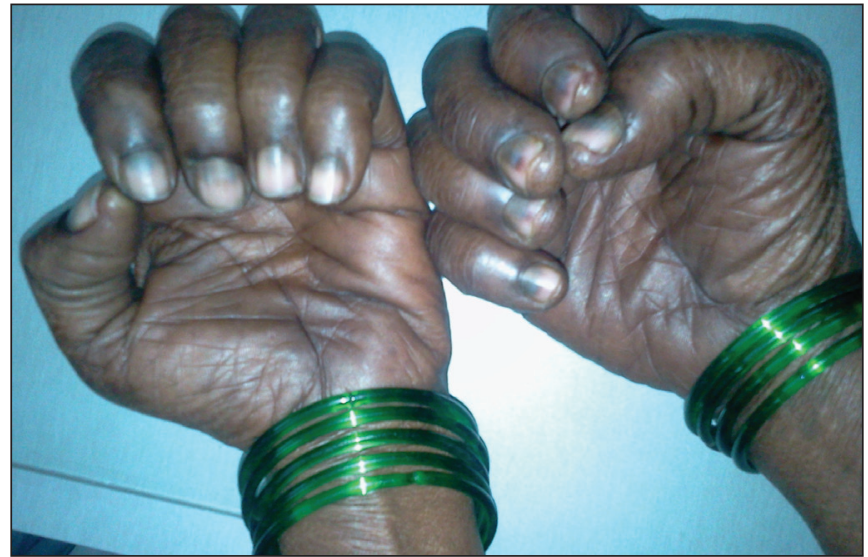

Fig. 1 Photograph shows melanonychia involving all fingernails, with hyperpigmentation of the nail beds and knuckles.

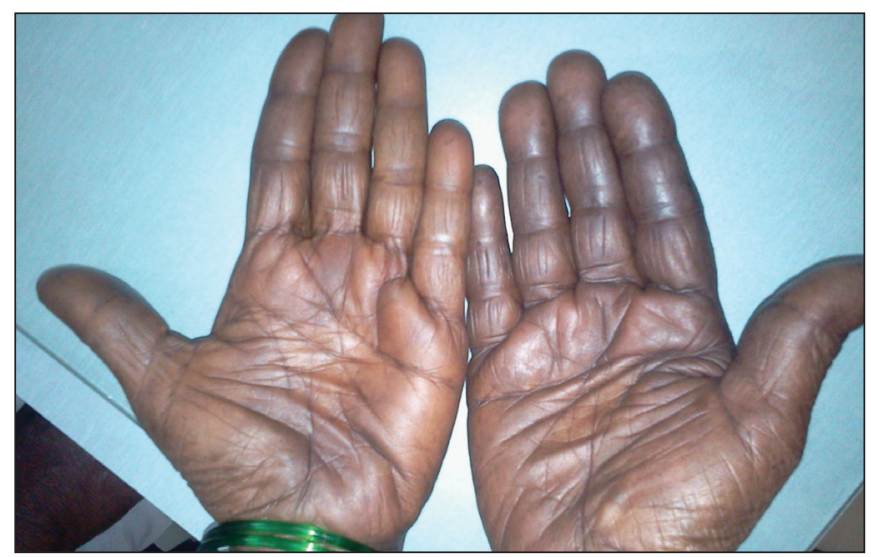

Fig. 2 Photograph shows irregular patches of hyperpigmentation involving the skin of the palmar surfaces of the hands.

${ }^{1}$ Department of Medicine, Kasturba Medical College, ${ }^{2}$ Department of Neurosurgery, Adarsha Superspeciality Hospital, Karnataka, India

Correspondence: Dr Suman S Karanth, Assistant Professor, Department of Medicine, Kasturba Medical College, \#17 OPD Block, Manipal University, Manipal, Karnataka, India.drsumansk@gmail.com 
(Fig. 1). There was no thickening or atrophy of the nails, and the adjacent skin was normal. Irregular hyperpigmented patches involving the dorsa of the feet and hands, the palmar surface of both hands (Fig. 2) and the perioral region were noted. She had not received any drugs known to cause hyperpigmentation, such as zidovudine, minocycline, cyclophosphamide and doxorubicin. Other causes implicated in hyperpigmentation such as vitamin B12 deficiency (vitamin B12 703 pg/mL; normal range 220-1,100 pg/mL), Addison's disease (cortisol $12.86 \mathrm{mcg} / \mathrm{dL}$; normal range $8-26 \mathrm{mcg} / \mathrm{dL}$ ), Cushing's syndrome, human immunodeficiency virus (negative enzyme-linked immunosorbent assays), scleroderma (negative for antinuclear antibodies), haemosiderosis (ferritin $67.14 \mathrm{ng} / \mathrm{mL}$; normal range $27-300 \mathrm{ng} / \mathrm{mL}$ ) and hyperthyroidism (thyroid-stimulating hormone $4.59 \mathrm{UIU} / \mathrm{mL}$; normal range 0.3-5 UIU/mL) were ruled out.

A diagnosis of hydroxyurea-induced hyperpigmentation was made. The acute onset of the hyperpigmentation following therapy lent support to the diagnosis. We temporarily reduced the patient's dosage of hydroxyurea to $500 \mathrm{mg}$ /day and awaited her next follow-up. The complete discontinuation of the drug was avoided as we feared that it might precipitate another thrombotic event.

\section{DISCUSSION}

Melanonychia due to hydroxyurea is very rarely reported when compared to skin hyperpigmentation..$^{(3)}$ It is more commonly seen among women. ${ }^{(1)}$ While the mechanism of melanonychia is not thoroughly understood, it is hypothesised to be due to photosensitisation and direct toxicity involving the nail matrix. A genetic predisposition is also thought to be contributory. ${ }^{(3)}$ Toxicity results in the stimulation of corresponding melanocytes and the deposition of melanin in the nail bed. ${ }^{(3)}$ Patterns of nail involvement present either as longitudinal or transverse bands, or diffuse hyperpigmentation. ${ }^{(1,2)}$ Longitudinal bands are the most common pattern seen, (1) and the thumb and index fingernails are the most frequently involved nails. Other less common patterns of cutaneous involvement include alopecia, leg ulceration, palmoplantar keratoderma and ichthyosis.(1) Teo and $\operatorname{Tan}^{(4)}$ reported the occurrence of melanonychia and skin hyperpigmentation in a patient as early as seven weeks after the initiation of hydroxyurea treatment, similar to that observed in our patient. Melanonychia has also been reported as late as five years after hydroxyurea therapy. ${ }^{(5)}$

In our patient, the fingernails were more involved than the toenails. This disparity has been attributed to the difference in the rate of nail growth of the hands and feet, with the toenails growing slower and therefore showing nail discolouration later. ${ }^{(6)}$ In our patient, the skin hyperpigmentation was not restricted to pressure points. It occurred over the palms, and the dorsa of the hands and feet. There was perioral hyperpigmentation as well. Such presentation has been described by Gropper et $\mathrm{al}_{;}^{(7)}$ the authors reported similar findings when treating polycythaemia vera using hydroxyurea. Interestingly, these authors also noted a gradual reduction in pigmentation despite the continuation of hydroxyurea at maximum dosage. When treating patients with melanonychia and hyperpigmentation, clinicians should take care to rule out closely mimicking conditions such as subungual melanoma, naevus, or pigmented squamous cell carcinoma.

In summary, there is a need for clinicians to be aware of the mucocutaneous side effects of hydroxyurea therapy. A correct diagnosis is essential to prevent undue fears in the patient. If necessary, therapy can be continued, even at maximum dosage, as mentioned. The decision to stop therapy needs to be carefully deliberated. It depends on the clinical setting and the physician's discretion after weighing the risk-benefit ratio.

\section{REFERENCES}

1. Aste N, Fumo G, Contu F, Aste N, Biggio P. Nail pigmentation caused by hydroxyurea: report of 9 cases. J Am Acad Dermatol 2002; 47:146-7.

2. Laughon SK, Shinn LL, Nunley JR. Melanonychia and mucocutaneous hyperpigmentation due to hydroxyurea use in an HIV-infected patient. Int J Dermatol 2000; 39:926-41.

3. Vomvouras S, Pakula AS, Shaw JM. Multiple pigmented nail bands during hydroxyurea therapy: an uncommon finding. J Am Acad Dermatol 1991; 24:1016-7.

4. Teo RY, Tan E. A case of hydroxyurea-induced transverse melanonychia. Int J Dermatol 2006; 45:1329-30.

5. Delmas-Marsalet B, Beaulieu P, Teillet-Thiebaud F, Jary L, Teillet F. Longitudinal melanonychia induced by hydroxyurea: four case reports and review of literature. Nouv Rev Fr Hematol 1995; 37:205-10.

6. Cakir B, Sucak G, Haznedar R. Longitudinal pigmented nail bands during hydroxyurea therapy. Int J Dermatol 1997; 36:236-7.

7. Gropper CA, Don PC, Sajadi MM. Nail and skin hyperpigmentation associated with hydroxyurea therapy for polycythemia vera. Int J Dermatol 1993; 32:731-3. 